\title{
Sitagliptin-balancing cardiovascular risk, hyperglycaemia and safety
}

A new study has shown that the oral dipeptidyl peptidase- 4 inhibitor sitagliptin increases insulin levels in patients with new-onset diabetes after transplantation (NODAT). The enzyme is involved in the degradation of incretins, which promote pancreatic insulin release.

Approximately $10-15 \%$ of renal transplant recipients develop NODAT, which is associated with increased risk of cardiovascular disease. "These patients have a very severe degree of arteriosclerosis against a background history of renal failure terminating in end-stage renal disease," explains study leader Trond Jenssen. "Treating their hyperglycaemia must not provoke further increases in cardiovascular risk or interfere with the immunosuppressant drugs being used and must be safe given the reduced renal function (single kidney)."

Typically, patients with NODAT are treated with either insulin or sulphonylurea agents. These treatments can lead to increases in body weight and in the frequency of hypoglycaemic episodes.

The study in 19 patients with NODAT showed that sitagliptin increased insulin secretion responses by $\geq 30 \%$ and significantly reduced fasting and $2-\mathrm{h}$ plasma glucose levels. No adverse effects were reported and endothelial function and markers of cardiovascular risk were unaffected, which bodes well for the future of the drug.

"Studies are needed to disclose the pathogenic mechanisms of NODAT to tailor the choice of therapy," concludes Jenssen.

Mina Razzak

Original article Strøm Halden, T. A. et al. Short-term efficacy and safety of sitagliptin treatment in long-term stable renal recipients with new-onset diabetes after transplantation. Nephrol. Dial. Transplant. doi:10.1093/ ndt/gft536 